\begin{tabular}{|c|c|c|}
\hline Beitr. Ent. & Keltern & ISSN 0005-805X \\
\hline $\mathbf{6 0}(2010) 1$ & S. $105-110$ & 31.07 .2010 \\
\hline
\end{tabular}

\title{
Bemerkungen zu der Gattung Elasmognathus FIEBER, 1844, und Beschreibung einer neuen Art
}

\section{(Hemiptera: Heteroptera: Tingidae)}

Mit 4 Figuren

Ursula GölLner-Scheiding

\section{Zusammenfassung}

Zu der Gattung Elasmognathus gehören die drei bekannten Arten auriculatus PÉRICART, 1985, und helferi Fieber, 1844, aus Asien sowie fieberi STÅL, 1855, aus Afrika, die kurz charakterisiert werden. Eine vierte neue Art aus Tansania (Afrika) wird als Elasmognathus tansaniellus beschrieben. Eine Bestimmungstabelle für die Arten und eine Literaturübersicht bilden den Abschluss der Arbeit.

\section{Summary}

The three known species of the genus Elasmognathus: auriculatus PÉRICART, 1985, and helferi FIEBer, 1844, from Asia and fieberi STÅL, 1855, out of Africa are charaterized shortly. One fourth new species is described from Tanzania (Africa) as Elasmognathus tansaniellus. A table for determination of the species is given and the literature is quoted.

\section{Einleitung}

In Heteroptera-Material aus Afrika (Tansania, Kenia und Rep. Südafrika), das mir freundlicherweise von J. Constant, Institut Royal des Sciences naturelles de Belgique, Départment d'Entomologie, zur Bearbeitung überlassen wurde, fand ich unter den Tingidae aus Tansania eine neue Art der Gattung Elasmognathus Fieber, 1844.

Die Gattung Elasmognathus wurde von FIEBER 1844 aufgestellt für die in „Ostindien“ von Dr. Helfer gefundene Art Elasmognathus helferi. Eine zweite Art der Gattung, Elasmognathus fieberi, ein Männchen, wurde dann von STÅL 1855 aus „Caffraria“ (Natal) beschrieben. Eine weitere neue Art, Elasmognathus auriculatus, konnte PÉRICART 1985 für Bhutan nachweisen. Die Gattung ist demnach in Afrika und im asiatischen Gebiet einschließlich der neuen Art mit jetzt insgesamt vier Arten verbreitet. Andere Arten, die der Gattung Elasmognathus zugeordnet wurden, stehen heute in den Gattungen Agachila und Paseala.

Dank der freundlichen Hilfe der Kustoden Dr. D. Burckhardt Museum Basel, Dr. G. Lindberg Museum Stockholm und Dr. H. ZetTel Museum Wien, konnte ich die Typen der bisher bekannten Arten der Gattung Elasmognathus überprüfen und möchte dafür Dank sagen. Zu danken habe ich auch Frau V. Heinrich und Herrn N. Hoff für die Anfertigung und Bearbeitung der Bilder. 


\title{
Bemerkungen zu bekannten Arten und Beschreibung einer neuen Art
}

\author{
Elasmognathus FIEBER, 1844: Abh.Böhm.Ges.Wiss., (5. Folge) 3, 368 (= Ent. \\ Monogr., 90).
}

\section{Typusart:}

Elasmognathus helferi Fieber, 1844: Abh.Böhm.Ges.Wiss., (5. Folge) 3, 369 (= Ent.Monogr., 91). (Monotypie).

Es handelt sich um längliche Tiere von 4,0 - etwa 5,5 mm Länge und 1,5 - 2 mm Breite von gelblicher bzw. gelblich-bräunlicher Färbung. Die Gattung ist auf Grund ihrer Pronotumgestaltung, das Pronotum weist bei allen Arten ohrmuschelartige Aufsätze auf, gut von anderen Gattungen abzugrenzen. Auch die vier Arten sind vor allem mit Hilfe dieser recht komplexen Bildungen des Pronotums zu trennen, hinzu kommen Unterschiede beispielsweise in der Breite des Costalrandes der Hemielytren. Während diese „Ohrmuscheln“ bei Elasmognathus helferi seitlich und kopfwärts relativ weit über die Schultern hinausragen, sind sie bei den anderen Arten seitlich und in Richtung Kopf weniger hervorstehend. Auch ist helferi mit etwa 5,5 mm Länge, im Vergleich mit den anderen Arten von etwa 4 - 4,5 mm Länge, die größte Art der Gattung.

$\mathrm{Zu}$ den bekannten zwei Arten aus Asien sind mit dem Fund der neuen Art aus Tansania jetzt auch für die Äthiopis zwei Arten der Gattung bekannt.

Die Verbreitung der Arten in Afrika und Indien spricht für ein relativ hohes Alter der Gattung und ihr Vorkommen noch vor der Trennung der Kontinente.

Als Wirt wird in einem Falle auf dem Etikett Cronton gratissima, eine Euphorbiaceae, angegeben.

\section{auriculatus PÉRICART, 1985: Ent. Brasil. 10: 49.}

\section{Typusart:}

HT Männchen, Bhutan, Samchi, 300-450 m, 7.-11.V.1972, expédition NHMB; NHM Basel. - 1 PT wie HT in Coll. PÉricart, 1 PT Männchen, Bhutan, Phuntsholing, 200-400 m, 25.IV.1972, expédition NHMB; HNMB.

\section{Verbreitung: Bhutan.}

Wirt: unbekannt.

Elasmognathus auriculatus ist eine Art von 4,5 - 4,6 mm Länge und gut 1,5 mm Breite. Sie ist etwas größer als fieberi, und die Hemielytren sind länger als bei dieser Art. Bisher sind von auriculatus nur Männchen bekannt. Die Art ist gekennzeichnet durch einen relativ breiten gewölbten oberen Rand der ohrmuschelartigen Bildungen des Pronotums. Die schmalen Verlängerungen der Ohrmuscheln stehen etwa ab Mitte relativ weit auseinander. In Bezug auf die Pronotumausbildung ist sie der afrikanischen Art Elasmognathus fieberi STÅL, 1855 ähnlich, jedoch sind die „Ohrmuscheln“ etwas höher und der obere Rand ist, wie gesagt, etwas breiter gewölbt. Auch ihr länglicher Fortsatz ist etwas breiter als bei fieberi. Ferner ist der Costalrand der Hemielytren bei auriculatus vorwiegend zweireihig, bei fieberi schmaler und einreihig. Bei der ebenfalls orientalisch verbreiteten Art helferi ist das Pronotum deutlich anders geformt und der Costalrand unregelmäßig mehrreihig. 
fieberi STÅL, 1855: Öfv. K. Vet. - Ak. Förh. 12 (1856), Nr. 1: 38.

\section{Typenmaterial:}

HT Männchen, „In terra Natalensi“ (Etikett: Caffria), J. A. WAhlberg; NHRS.

Verbreitung: Rep. Südafrika.

Wirt: unbekannt.

Es handelt sich um die kleinste Art der Gattung aus der Äthiopis von $4 \mathrm{~mm}$ Länge und 1,5 mm Breite von hellbräunlicher Färbung; allerdings ist mir nur der Typus, ein Männchen, bekannt. Die neue afrikanische Art ist größer und heller gefärbt als die bekannte Art fieberi, auch ist der Bau der Ohrmuscheln unterschiedlich. So ist der Rand der Wölbung bei fieberi breiter und die Öffnung zeigt mehr nach hinten, bei der neuen Art ist der Rand relativ schmal und mehr nach oben geöffnet. Der Costalrand der Hemielytren ist bei fieberi einreihig, bei allen anderen Arten mehrreihig.

helferi Fieber, 1844: Abh. Böhm. Ges. Wiss., (5. Folge) 3, 369 (= Ent. Monogr. 91).

\section{Typenmaterial:}

HT Weibchen, „Ostindien“ (Etikett: Indes or.), Dr. Helfer; NHMW.

Verbreitung: Indien.

Wirt: unbekannt.

Elasmognathus helferi ist mit 5,5 mm Länge die größte Art der Gattung, gefangen wurde sie in „Ostindien“. Mir ist nur der Typus, ein Weibchen, bekannt. Er ist von hellbrauner Färbung mit etwas dunkleren Ohrmuscheln, sollte jedoch nach der Beschreibung von Fieber „gelblichweiss ins Bräunliche ziehend“ gefärbt sein. Die großen Ohrmuscheln überragen seitlich weit den Körper, besitzen einen relativ schmalem gewölbten Rand und sind nach oben geöffnet. Die Pronotumauflage ist nur kurz im oberen Teil in der Mitte verbunden und der Mittelkiel daher gut sichtbar, der Costalrand der Hemielytren ist unregelmäßig zweireihig.

\section{tansaniellus nov. spec.}

\section{Typenmaterial:}

HT Weibchen, Tanzania: Tanga Muheza Dist. Kwamgumi, For Res 457'S 3844'E, 170-220 m, 22.VII.1995, Fog 9 CM, Zmuc Denmark; ISNB. - Paratypen 2 Weibchen: Tanzania: Tanga Muheza, Dist. Kwamgumi, For Res 45⒌'S 3844'E, 170-220 m, 23.VII.1995, Fog 10 BO, Zmuc Denmark; ISNB - Tanzania: Tanga Muheza Dist. Kwamgumi, For Res $4^{\circ} 57^{\prime} \mathrm{S} 38^{\circ} 44^{\prime} \mathrm{E}$, 170-220 m, 23.VII.1995, Fog 10 AL, Zmuc Denmark; MNB.

Verbreitung: Rep. Südafrika, Tansania.

Wirt: Croton gratissima (Euphorbiaceae).

Die neue Art ist einfarbig gelblich gefärbt und von etwa 4,5 mm Länge und $2 \mathrm{~mm}$ Breite, Gefangen wurden drei Weibchen mit Hilfe eines Nebelgerätes. Es ist daher anzunehmen, dass die Art auf Bäumen lebt und daher nicht leicht nachzuweisen ist. 
Ein weiteres, leider stark beschädigtes Exemplar befindet sich im Museum Tervuren, gesammelt am 4. I.[19]64 in Warmbaths, Transvaal (Rep. Südafrika), leg. Al. Capener. Irrtümlicherweise wurde es als Elasmognathus fieberi STÅL bestimmt.

Der Kopf ist kurz und mit fünf Dornen unterschiedlicher Länge besetzt, die Wangenplatten überragen den Kopf vorn nur wenig, die Augen sind dunkel. Die Antennen sind gelblich mit einem dunkel gefärbten und behaarten vierten Endglied; das Längenverhältnis der Glieder zueinander beträgt $1: 1,2: 6.5: 2$. Das Rostrum ist kurz und reicht knapp bis zu den Coxen des zweiten Beinpaares, es liegt in einer Rinne. Die Beine sind schlank, gelblich gefärbt mit einzelnen dunkleren Flecken und mit dunklen Tarsen, die hinteren beiden Beinpaare stehen weit auseinander. Die Pronotumfläche ist, soweit sichtbar, fein punktiert und läuft vorn in eine hoch gebogene Spitze aus, die ohrmuschelartige Auflage ist in Maschen unterteilt und hoch gerundet mit einem schmalen gewölbten Rand, seitlich bis etwa Körperbreite überstehend, die relativ große Öffnung ist nach oben gerichtet. Die schmalen etwas gewölbten Fortsätze der Auflage lassen einen kleinen Zwischenraum frei und laufen hinten zusammen, nur durch die deutliche kielartige Mittellinie getrennt, diese vom Vorderrand des Pronotums bis zur Endspitze durchlaufend, die beiden seitlichen Linien sind nur am unteren Ende sichtbar. Die Hemielytren überragen den Körper und sind von gelblicher Färbung, in einem Falle ist der Costalrand etwa in der Mitte braun gefleckt, der Costalrand ist relativ breit zweireihig mit unregelmäßigen Maschen und überragt den Körper seitlich, die Subcostalfläche ist schmal zweireihig, die Discoidalfläche mit relativ kleinen Maschen ist bis zu neun Reihen breit, die Suturalfläche ist im vorderen Abschnitt kleinmaschig, im hinteren Teil großmaschiger. Die Ventralseite ist gelblich gefärbt, der Thorax ist punktiert und die Drüsenöffnungen sind gut erkennbar.

\section{Bestimmungstabelle}

1 Ohrmuschelartige Bildungen des Pronotums weniger groß und den Körper seitlich weniger deutlich überragend, Costalrand der Hemielytren unterschiedlich breit, kleinere Tiere von $4-4,6$ mm Länge, in der äthiopischen und der orientalischen Region vorkommend, von gelblich-bräunlicher Färbung.

2

1* Ohrmuschelartige Bildungen des Pronotums größer und den Körper seitlich weit überragend, größere Art von 5,5 mm Länge, Costalrand der Hemielytren mehrreihig, hellbraun gefärbt. In „Ostindien“ und Philippinen (Luzon) vorkommend. ............... helferi Fieber

2 Im äthiopischen Faunengebiet gefunden. .. 3

2* Vorkommen im asiatischen Faunengebiet (Bhutan), Costalrand der Hemielytren mehrreihig, obere Wölbung der „Ohrmuschel“ relativ breit und stark gewölbt, Öffnung mehr seitlich gerichtet, von 4,5-4,6 mm Länge. auriculatus PÉRICART

3 Costalrand der Hemielytren einreihig, obere Wölbung der „Ohrmuschel“ relativ breit, deutlich gewölbt, Öffnung kleiner und nach hinten gerichtet, kleine Art von 4 mm Länge. In der Rep. Südafrika vorkommend. fieberi Stål

$3^{*} \quad$ Costalrand der Hemielytren mehrreihig, obere Wölbung der „Ohrmuschel“ schmal und mit größerer Öffnung, diese nach oben gerichtet, von gelblicher Färbung, von 4,5 mm Länge. In Tansania und Rep. Südafrika gefunden. . tansaniellus $\mathrm{n} . \mathrm{sp}$. 
Beitr. Ent. 60 (2010) 1

\section{Abkürzungen der Museen}

HNMB Naturhistorisches Museum Basel, Schweiz

ISNB Institut Royal des Sciences Naturelles de Belgique, Brüssel, Belgien

MNB Museum für Naturkunde Berlin, Deutschland

NHRS Naturhistoriska Riksmuseet Stockholm, Schweden

\section{Literatur}

Drake, C. J. 1927: Tingitidae from the Far East and Madagascar (Hemiptera). - The Philippine Journal of Sciences 34: 307-312.

Fieber, F. X. 1844: Entomologische Monographien. - Abhandlungen der k. böhmischen Gesellschaft der Wissenschaften (V. Folge) 3: 277-416, 1845 (= Entomologische Monographien: 137 S., 1844).

PérICART, J. 1985: Tingidae nouveaux ou intéressants du Nord du sous-continent Indien (Hemiptera). II. - Entomologica Brasiliensia 10: 27-62.

SтĂL, C. 1855: Hemiptera fran Kafferlandet. - Öfversigt af Kongliga Svenska Vetenskap-Akademiens Förhandlingar 12 (1856): 27-46.

Anschrift der Verfasserin:

Dr. URSUla GölLnER-SCHEIDING

Museum für Naturkunde, Leibniz-Institut für

Evolutions- und Biodiversitätsforschung an der

Humboldt-Universität zu Berlin

Invalidenstr. 43

10115 Berlin

Deutschland

\section{Subject editor:}

Dr. J. Deckert 


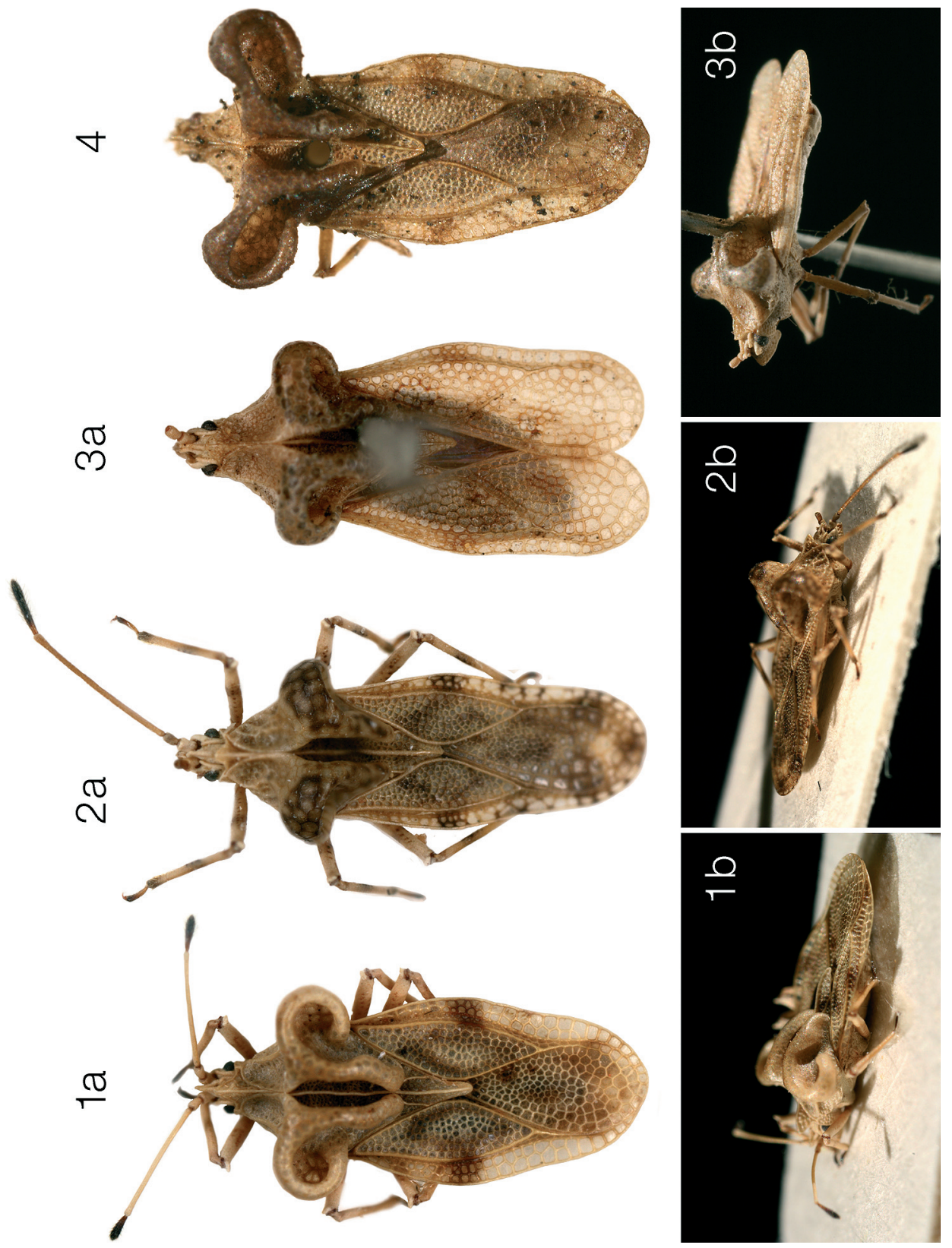

Fig. 1-4: 1a. Elasmognathus tansaniellus spec. nov., dorsal; 1b. seitlich;

2a. Elasmognathus auriculatus PÉRICART, 1985, dorsal; 2b. seitlich;

3a. Elasmognathus fieberi STÅL, 1855, dorsal; 3b. seitlich;

4. Elasmognathus helferi Fieber, 1844. 